\title{
Lymphocyte-rich classical Hodgkin's lymphoma: distinctive tumor and microenvironment markers
}

\author{
Syong H Nam-Cha ${ }^{1,2}$, Santiago Montes-Moreno ${ }^{2}$, Maria T Salcedo ${ }^{3}$, Josefina Sanjuan ${ }^{4}$, \\ Juan F Garcia ${ }^{2,5}$ and Miguel A Piris ${ }^{2}$ \\ ${ }^{1}$ Department of Pathology, Complejo Hospitalario Universitario de Albacete, Albacete, Spain; ${ }^{2}$ Lymphoma \\ Group, Molecular Pathology Programme, Spanish National Cancer Research Centre (CNIO), Madrid, Spain; \\ ${ }^{3}$ Department of Pathology, Hospital Universitario Vall d' Hebron, Barcelona, Spain; ${ }^{4}$ Department of \\ Pathology, Hospital Arnau de Vilanova, Valencia, Spain and ${ }^{5}$ Department of Pathology, MD Anderson \\ International Spain, Madrid, Spain
}

\begin{abstract}
The existence, diagnostic features, and the biological and clinical relevance of lymphocyte-rich classical Hodgkin's lymphoma remain controversial. A comparative marker analysis of lymphocyte-rich classical Hodgkin's lymphoma, nodular lymphocyte-predominance Hodgkin's lymphoma, and of other subtypes of classical Hodgkin's lymphoma was carried out. Markers were selected focusing on B-cell lineage and transcription program (OCT.1, ОСТ.2, ВOB.1, BCL6, PAX-5, GCET1, KLHL6, and BLIMP1), the NF-KB signaling pathway (REL-B, C-REL, TRAF-1, p-50, and MUM-1) and the T-cell microenvironment (CD3, CD57, PD-1, CXCL13, and CD10, BCL-6, CD23). Lymphocyte-rich classical Hodgkin's lymphoma cases displayed features intermediate between those of classical Hodgkin's lymphoma and nodular lymphocyte-predominance Hodgkin's lymphoma. The expression of B-cell transcription factors such as OCT.1, OCT.2, BOB.1, and BCL6 was more frequent in lymphocyte-rich classical Hodgkin's lymphoma than in classical Hodgkin's lymphoma. A follicular T-cell microenvironment was also identified in 50\% of lymphocyte-rich classical Hodgkin's lymphoma cases. NF-kB markers were expressed at frequencies comparable with those observed in classical Hodgkin's lymphoma. The neoplastic cell immunophenotype and microenvironment in lymphocyte-rich classical Hodgkin's lymphoma closely mimic that which are observed in the outer zone of the germinal center, where B-cell blasts with germinal-center markers co-express CD30 and the B-cell transcription program, surrounded by follicular T-cell rosettes. Lymphocyte-rich classical Hodgkin's lymphoma seems to be characterized by a stronger expression of the B-cell transcription program by the neoplastic cells and by a follicular T-cell background, occupying an intermediate position between classical Hodgkin's lymphoma and nodular lymphocyte-predominance Hodgkin's lymphoma.

Modern Pathology (2009) 22, 1006-1015; doi:10.1038/modpathol.2009.54; published online 22 May 2009
\end{abstract}

Keywords: lymphocyte-rich classical Hodgkin's lymphoma; T-cell microenviroment

Hodgkin's lymphoma has been subclassified according to the World Health Organization classification into two different types: classical Hodgkin's lymphoma and nodular lymphocyte-predominant Hodgkin's lymphoma. ${ }^{1}$ Classical Hodgkin's lymphoma itself may be subdivided into four diagnostic subtypes: nodular sclerosis, mixed cellularity, lymphocyte-rich, and lymphocyte-depleted Hodgkin's

Correspondence: Dr SH Nam-Cha, MD, Molecular Pathology Programme, Centro Nacional de Investigaciones Oncológicas, C/ Melchor Fernández Almagro 3, Madrid E-28029, Spain.

E-mail: tnamcha@yahoo.es

Received 28 January 2009; revised 2 March 2009; accepted 3 March 2009; published online 22 May 2009 lymphoma. Lymphocyte-rich classical Hodgkin's lymphoma accounts for only $3-5 \%$ of all Hodgkin's lymphomas., ${ }^{1,2}$ Most lymphocyte-rich classical Hodgkin's lymphomas are diagnosed at early stages and have a better prognosis than do other classical Hodgkin's lymphomas, being more similar to nodular lymphocyte-predominant Hodgkin's lymphoma. ${ }^{2}$ Lymphocyte-rich classical Hodgkin's lymphoma is characterized histologically by a small number of Reed-Sternberg cells, distributed at the edge of reactive follicles, with a classical Hodgkin's lymphoma immunophenotype.

Histological and clinical features of lymphocyterich classical Hodgkin's lymphoma have been debated, and there is no clear consensus on whether 
lymphocyte-rich classical Hodgkin's lymphoma represents a distinct disorder or just an early presentation of classical Hodgkin's lymphoma. ${ }^{3}$

In this study, we have carried out a comparative marker analysis of lymphocyte-rich classical Hodgkin's lymphoma, nodular lymphocyte-predominant Hodgkin's lymphoma, and other classical Hodgkin's lymphoma cases, interrogating panels of immunomarkers selected for the study of the B-cell lineage and transcription program, the $\mathrm{NF}-\mathrm{\kappa B}$ signaling pathway, and the T-cell microenvironment. We have additionally investigated whether the outer zone of the germinal center could be a normal counterpart of lymphocyte-rich classical Hodgkin's lymphoma, sharing the immunophenotype of neoplastic cells and their microenvironment.

\section{Materials and methods}

We obtained 143 cases diagnosed earlier as nodular sclerosis classical Hodgkin's lymphoma, mixed cellularity classical Hodgkin's lymphoma, lymphocyte-rich classical Hodgkin's lymphoma, or nodular lymphocyte-predominant Hodgkin's lymphoma from the files of the Molecular Pathology Programme of the Spanish National Cancer Centre (CNIO), Madrid, Spain, under the supervision of the local ethical committee. All cases were reviewed and diagnosed according to the criteria of the World Health Organization ${ }^{1}$ classification. The cases were grouped into three diagnostic categories: lymphocyte-rich classical Hodgkin's lymphoma $(n=16)$, nodular lymphocyte-predominant Hodgkin's lymphoma $(n=68)$, and other types of classical Hodgkin's lymphoma $(n=59)$. On the basis of these categories, three tissue microarray blocks were constructed using a Tissue Arrayer Device (Beecher Instrument, Silver Spring, MD, USA), as described earlier. ${ }^{4}$ All the tissue microarray block sections were immunostained with a panel of antibodies against CD30 (CON6D/B9, CNIO Monoclonal Antibody Unit, Madrid, Spain), CD20 (L26, DAKO, Glostrup, Denmark), CD15 (153A/D6, CNIO Monoclonal Antibody Unit), OCT.1 (12F11, Santa Cruz Biotechnology, Santa Cruz, CA, USA), OCT.2 (C-20 polyclonal, Santa Cruz Biotechnology), BOB.1 (polyclonal, Santa Cruz Biotechnology), bcl-6 (GI191E/ A8, CNIO Monoclonal Antibody Unit), PAX-5 (polyclonal, Santa Cruz Biotechnology), GCET-1 (341B/C1, CNIO Monoclonal Antibody Unit), KLHL6 (92C/D9, CNIO Monoclonal Antibody Unit), MUM-1 (ICSAT/M-17, Santa Cruz Biotechnology), C-REL (B-6, Santa Cruz Biotechnology), REL-B (polyclonal, Santa Cruz Biotechnology), TRAF-1 (H-3, Santa Cruz Biotechnology), p-50 (polyclonal, Gene Tex, San Antonio, TX, USA), IgD (polyclonal, DAKO), BLIMP-1 (195G/G5, CNIO Monoclonal Antibody Unit), CD38 (VS38, DAKO), CD3 (polyclonal, DAKO), CD57 (B321/NK-1, Abcam, Cambridge, UK), PD-1 (NAT105, Abcam ${ }^{5}$ ), CD10 (56C6; Novocastra,
Newcastle-upon-Tyne, UK), and CXCL13 (53610, R\&D Systems, Minneapolis, MN, USA), using heatinduced epitope retrieval, as described earlier. Nonisotopic in situ hybridization for the Epstein-Barr virus (EBV) early RNA (EBER) was carried out using a fluorescein-conjugated PNA probe (DAKO) and an anti-FITC antibody (clone DAK-FITC4, DAKO), following the manufacturer's recommendations. Staining with CD23 (MHM6, DAKO) was carried out in whole sections of 15 lymphocyte-rich classical Hodgkin's lymphoma cases. Double immunohistochemical or immunofluorescence staining with PD-1 and CD30, bcl-6 and CD30, OCT.2 and CD30, and BOB.1 and CD30 was also carried out in reactive tonsil, reactive lymph nodes, and in six lymphocyterich classical Hodgkin's lymphoma cases, in which preserved residual germinal centers could be easily identified, using protocols described earlier. ${ }^{5,6}$ Immunofluorescence slides were examined on a Nikon E800 Eclipse fluorescence microscope (Nikon, Kingston-upon-Thames, UK) equipped for epifluorescence, and images were captured with an Axiocam charge-coupled device (CCD) camera (Zeiss, Jena, Germany) and Axiovision software (Imaging Associates, Bicester, UK), and adjusted using Photoshop software (Adobe, San Jose, CA, USA).

Statistical analyses, conducted using SPSS 12.0, consisted of $\chi^{2}$ tests to compare frequencies of the three different diagnostic groups of each of the immunomarkers. Significance was concluded for values of $P<0.05$.

\section{Results}

Results are presented for the diagnostic groups, and separately for the tumoral cell phenotype and T-cell microenvironment.

\section{Tumoral Cell Phenotype}

The main features related to the tumoral cell phenotype are summarized in Table 1 and illustrated in Figures 1 and 2.

\section{Classical Hodgkin's lymphoma}

Mononuclear Hodgkin's cells and multinucleated Reed-Sternberg cells were CD30 positive in all cases, whereas a CD15 expression was seen in 52\% of cases. Seven percent of cases were CD20 weakly positive, whereas an EBER expression in the mononuclear Hodgkin's cells and multinucleated ReedSternberg cells was detected in $29 \%$ of cases.

Regarding the B-cell lineage and transcription program, OCT.1, OCT.2 and their coactivator BOB.1 were expressed by mononuclear Hodgkin's cells and multinucleated Reed-Sternberg cells only in 21, 34, and $26 \%$ of cases, respectively. All cases were Bcl-6 negative. Moreover, mononuclear Hodgkin's cells and multinucleated Reed-Sternberg cells expressed Pax-5 (moderate to strong staining) in $80 \%$ of the 
Table 1 Summary of the immunohistochemical findings

\begin{tabular}{lccc}
\hline & \multicolumn{3}{c}{ Percentage positive cases (positive/valuable cases) } \\
\cline { 2 - 4 } Antibody & CHL $(n=59)$ & LRCHL $(n=16)$ & NLPHL $(n=68)$ \\
\hline CD30 & $100(59 / 59)$ & $100(16 / 16)$ & $0(0 / 68)$ \\
CD20 & $7(4 / 58)$ & $31(5 / 16)$ & $98(48 / 49)$ \\
CD15 & $52(31 / 59)$ & $56(9 / 16)$ & $4(6 / 68)$ \\
EBER & $29(17 / 58)$ & $31(5 / 16)$ & $1(1 / 57)$ \\
OCT.1 & $21(12 / 58)$ & $50(8 / 16)$ & $69(42 / 61)$ \\
OCT.2 & $34(20 / 58)$ & $56(9 / 16)$ & $100(54 / 54)$ \\
BOB.1 & $26(15 / 58)$ & $62(10 / 16)$ & $97(62 / 64)$ \\
Bcl-6 & $0(0 / 55)$ & $31(5 / 16)$ & $82(51 / 62)$ \\
Pax-5 & $80(45 / 56)$ & $94(15 / 16)$ & $94(60 / 64)$ \\
GCET-1 & $21(11 / 52)$ & $0(0 / 16)$ & $74(44 / 59)$ \\
KLHL6 & $40(23 / 57)$ & $69(11 / 16)$ & $78(47 / 60)$ \\
MUM-1 & $100(58 / 58)$ & $100(16 / 16)$ & $20(13 / 64)$ \\
c-Rel & $60(35 / 58)$ & $25(4 / 16)$ & $20(12 / 59)$ \\
Rel-B & $54(31 / 57)$ & $12(2 / 16)$ & $40(22 / 55)$ \\
TRAF1 & $95(54 / 57)$ & $100(16 / 16)$ & $19(14 / 57)$ \\
p-50 & $85(46 / 54)$ & $73(11 / 15)$ & $36(22 / 61)$ \\
IgD & $0(0 / 59)$ & $6(1 / 16)$ & $17(10 / 60)$ \\
Blimp-1 & $27(16 / 58)$ & $25(4 / 16)$ & $50(27 / 54)$ \\
CD38 & $10(6 / 58)$ & $0(0 / 16)$ & $9(6 / 63)$ \\
& & &
\end{tabular}

cases, and $40 \%$ expressed the BTB-kelch protein, KLHL6. The recently described GCET1 (germinalcenter B-cell-expressed transcript-1) was observed in $21 \%$ of cases. Six of the 58 cases expressed CD38 $(10 \%)$ and Blimp-1 was observed in 16 of the 58 cases (27\%). All cases were IgD negative.

With respect to the NF- $\mathrm{KB}$ signaling pathway, c-Rel and Rel-B nuclear staining in mononuclear Hodgkin's cells and in multinucleated Reed-Sternberg cells was observed in 60 and $54 \%$ of cases, respectively. TRAF1 and p-50 were detected in mononuclear Hodgkin's cells and in multinucleated Reed-Sternberg cells in 95 and $85 \%$ of the cases, respectively. All cases were MUM-1 positive.

\section{Nodular lymphocyte-predominant Hodgkin's lymphoma}

Lymphocytic and/or histiocytic Reed-Sternberg cell variants were CD20 positive in $98 \%$ of the cases, CD30 was consistently negative, and CD15 was expressed in 6 out of 68 cases (4\%). EBER was only detected in one case.

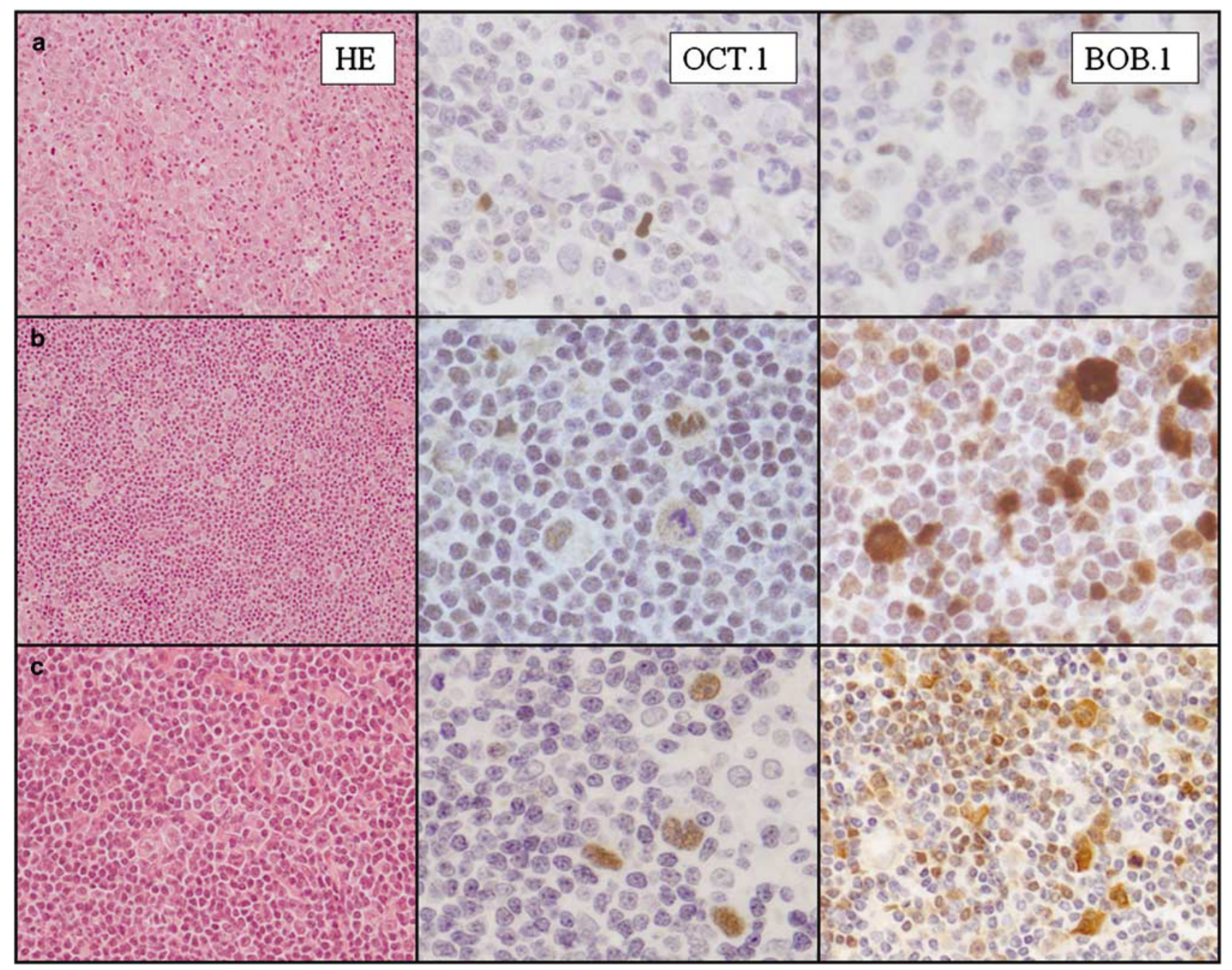

Figure 1 Expression of OCT.1 and BOB.1 in CHL (a), LRCHL (b), and NLPHL cases (c). 


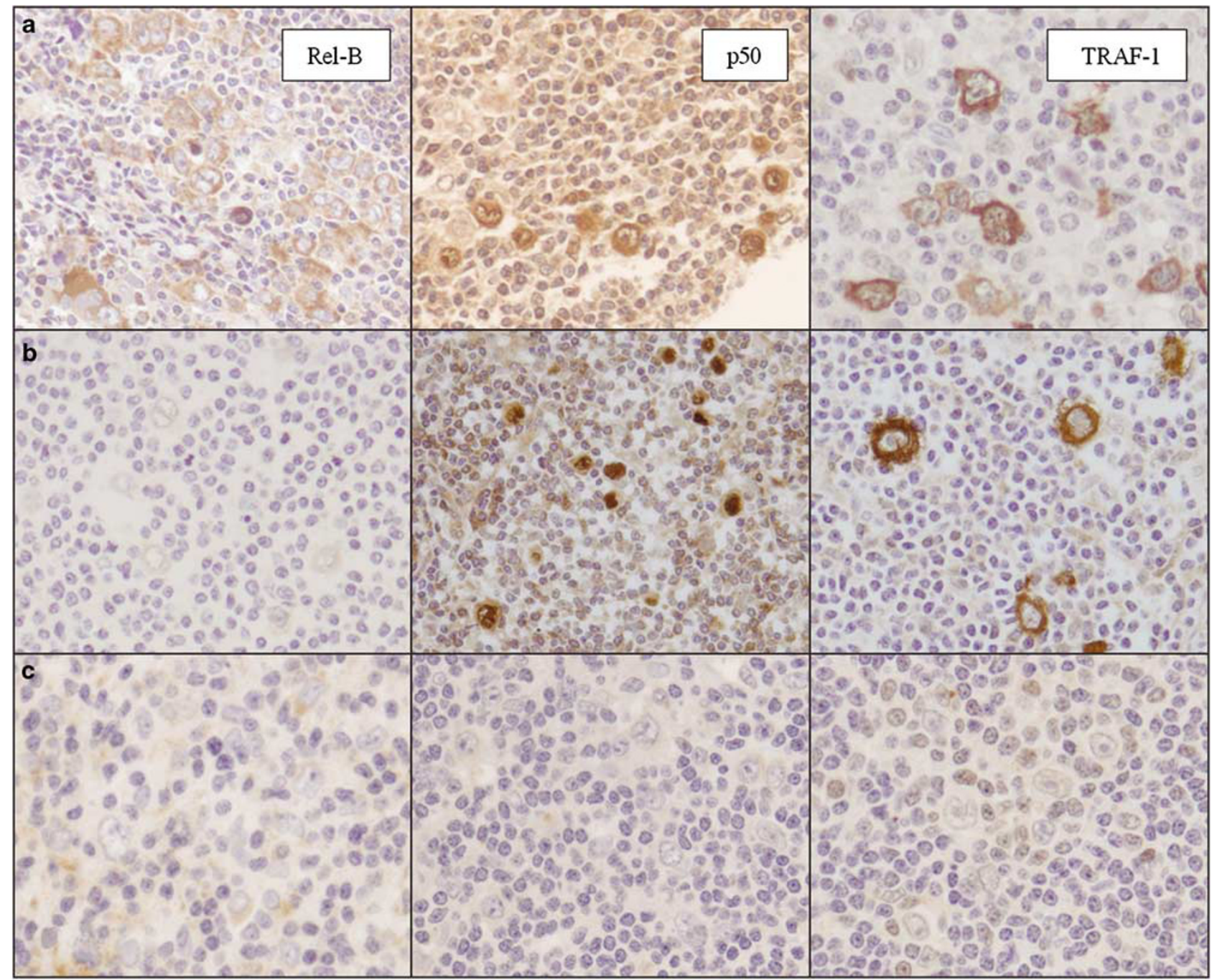

Figure 2 Expression of Rel-B, p-50, and TRAF-1 in CHL (a), LRCHL (b), and NLPHL cases (c), respectively.

In this group, OCT.2 was expressed in all cases, BOB1 in $97 \%$, and OCT.1 in $69 \%$ of the cases. Other B-cell lineage markers such as bcl-6, Pax-5, KLHL6, and GCTE-1 were detected in $82,94,78$, and $74 \%$ of the cases, respectively. With regard to plasma cell differentiation, Blimp-1 was detected in $50 \%$ of cases, but IgD and CD38 were only shown in 10 of 60 $(17 \%)$ and in 6 of $63(10 \%)$ cases, respectively.

Nuclear expression of NF- $\mathrm{BB}$ subunits in nodular lymphocyte-predominant Hodgkin's lymphoma was rarely seen. Staining was observed for c-Rel in only $20 \%$, for Rel-B in $40 \%$, for TRAF1 in $19 \%$, and for p50 in $36 \%$ of cases. MUM-1 staining was observed in $20 \%$ of the cases.

\section{Lymphocyte-rich classical Hodgkin's lymphoma}

All the cases diagnosed as lymphocyte-rich classical Hodgkin's lymphoma had classical CD30-positive mononuclear Hodgkin's cells and a multinucleated Reed-Sternberg immunophenotype. They were pre- dominantly CD15 positive (56\%) and $31 \%$ of cases were EBER positive. Pax-5 was expressed in $94 \%$ of cases. CD20 was observed in $31 \%$ of cases, which was more frequent than that in classical Hodgkin's lymphoma, but not significantly so.

Interestingly, immunomarkers related to the B-cell transcription program were identified: OCT.1 was expressed in $50 \%$, OCT. 2 in $56 \%$, and BOB. 1 in $62 \%$ of cases. Germinal-center immunomarkers were also detected (Bcl-6 in 36\% and KLHL6 in 69\%), except for GCET-1, which was constantly negative.

The expression of NF-кB subunits was variable. CRel and Rel-B were expressed in 25 and $12 \%$ of cases, respectively. Nevertheless, TRAF-1 and p-50 (NF-кB1) (canonical pathway) were observed in 100 and $73 \%$ of the cases, respectively. MUM-1 was positive in all cases.

IgD and Blimp-1 were only observed in $1(6 \%)$ and $4(25 \%)$ of the 16 cases, respectively. CD38 was constantly negative. 


\section{T-Cell Microenvironment}

The main features related to the T-cell microenvironment are summarized in Table 2 and illustrated in Figure 3.

\section{Nodular lymphocyte-predominant Hodgkin's lymphoma}

A total of $77 \%$ of the nodular lymphocyte-predominant Hodgkin's lymphoma cases showed distinct

Table 2 Immunophenotype of T-cell rosettes

Percentage positive cases (positive/valuable cases)

\begin{tabular}{lrcc} 
Antibody & CHL $(n=59)$ & LRCHL $(n=16)$ & NLPHL $(n=68)$ \\
\hline CD3 & $17(0 / 57)$ & $62.5(10 / 16)$ & $55.22(37 / 67)$ \\
CD57 & $0(0 / 55)$ & $20(3 / 15)$ & $77.19(44 / 67)$ \\
PD-1 (Nat-105) & $0(0 / 52)$ & $53.33(8 / 15)$ & $87.69(57 / 65)$ \\
CXCL-13 & $0(0 / 54)$ & $12.5(2 / 16)$ & $12.31(8 / 65)$ \\
Bcl-6 & $0(0 / 59)$ & $0(0 / 16)$ & $0(0 / 68)$ \\
CD-10 & $0(0 / 59)$ & $0(0 / 16)$ & $0(0 / 68)$
\end{tabular}

CD57-positive rosettes surrounding tumoral cells. The rosettes were also apparent with PD-1 (NAT$105)$ in a higher proportion of cases (88\%) compared with CD57. These T-cell rosettes were also identified with CD3 in $55 \%$ of the cases. Moreover, eight cases showed CXCL-13-positive T-cell rosettes. No bcl-6-positive or CD10-positive T-cell rosettes were observed.

\section{Classical Hodgkin's lymphoma}

No follicular T-cell rosettes surrounding mononuclear Hodgkin's cells and multinucleated ReedSternberg cells could be identified in this diagnostic group with either of the immunomarkers, except in CD3, which yielded T-cell rosettes in 10 cases $(17 \%)$.

Lymphocyte-rich classical Hodgkin's lymphoma Cases diagnosed as lymphocyte-rich classical Hodgkin's lymphoma displayed follicular T-cell rosettes surrounding tumoral mononuclear Hodgkin's cells and multinucleated Reed-Sternberg cells. With

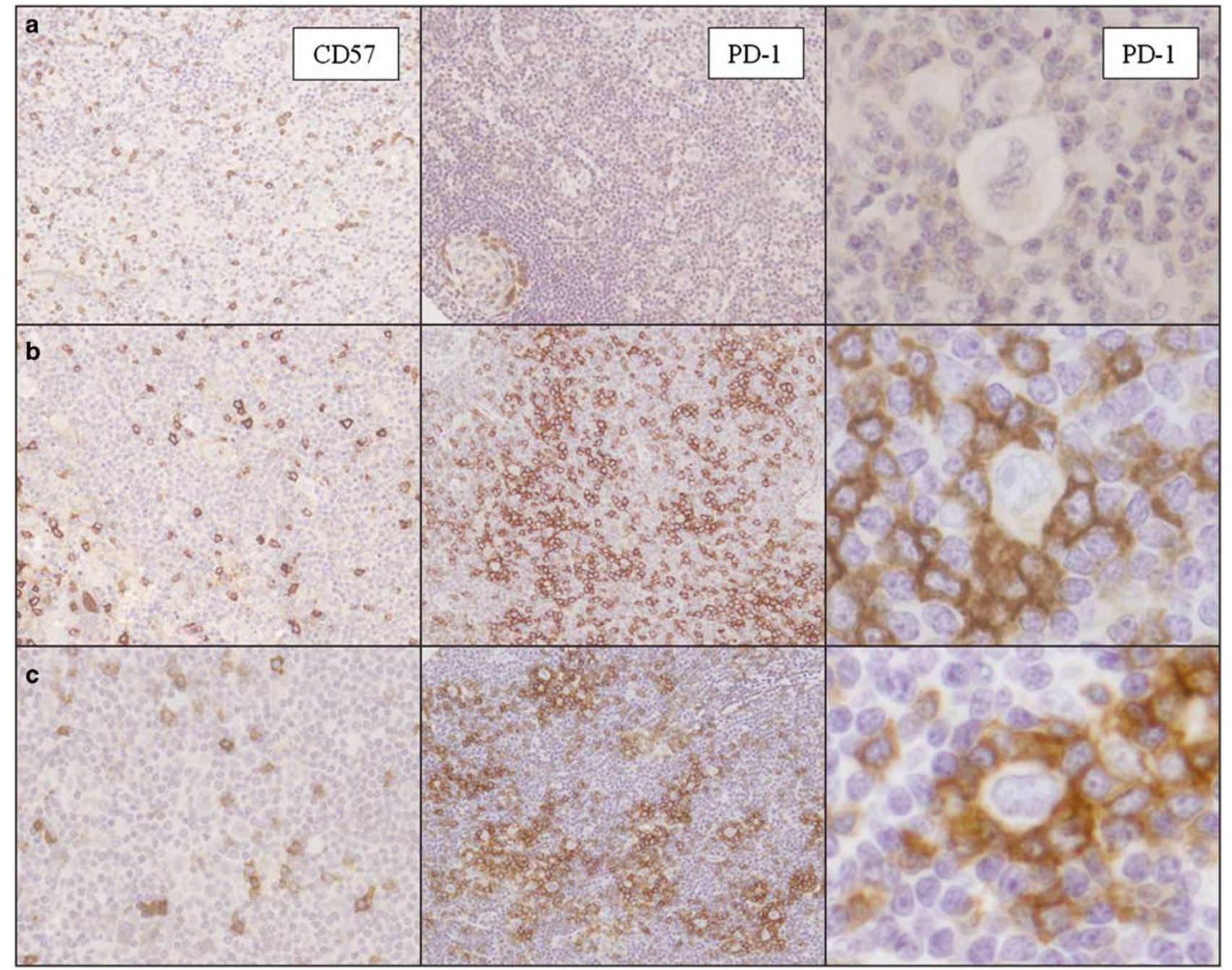

Figure 3 Follicular T-cell rosettes in CHL (a), LRCHL (b), and NLPHL cases (c) with CD57 and PD-1. 
Table 3 Statistical analysis (NS = not significant)

\begin{tabular}{lccc}
\hline Tumoral cell & CHL vs & $\begin{array}{c}\text { LRCHL vs } \\
\text { NLPHL }\end{array}$ & $\begin{array}{c}\text { NLPHL vs } \\
\text { CHL }\end{array}$ \\
\hline CD30 & NS & $P<0.05$ & $P<0.05$ \\
CD20 & NS & $P<0.05$ & $P<0.05$ \\
CD15 & NS & $P<0.05$ & $P<0.05$ \\
EBER & NS & $P<0.05$ & $P<0.05$ \\
OCT.1 & $P<0.05$ & NS & $P<0.05$ \\
OCT.2 & NS & $P<0.05$ & $P<0.05$ \\
BOB.1 & $P<0.05$ & $P<0.05$ & $P<0.05$ \\
Bcl-6 & $P<0.05$ & $P<0.05$ & $P<0.05$ \\
Pax-5 & NS & NS & $P<0.05$ \\
GCET-1 & $P<0.05$ & $P<0.05$ & $P<0.05$ \\
KLHL6 & $P<0.05$ & NS & $P<0.05$ \\
MUM-1 & NS & $P<0.05$ & $P<0.05$ \\
c-Rel & $P<0.05$ & NS & $P<0.05$ \\
Rel-B & $P<0.05$ & $P<0.05$ & NS \\
TRAF1 & NS & $P<0.05$ & $P<0.05$ \\
p-50 & NS & $P<0.05$ & $P<0.05$ \\
IgD & NS & NS & $P<0.05$ \\
Blimp-1 & NS & NS & $P<0.05$ \\
CD38 & NS & NS & NS \\
& & & \\
T-cell rosettes & & NS & $P<0.05$ \\
CD3 & $P<0.05$ & $P<0.05$ & $P<0.05$ \\
CD57 & $P<0.05$ & $P<0.05$ & $P<0.05$ \\
PD-1(Nat-105) & $P<0.05$ & NS & $P<0.05$ \\
CXCL-13 & $P<0.05$ & NS & NS \\
Bcl-6 & NS & & NS \\
CD-10 & NS & & \\
\hline & & & \\
\hline
\end{tabular}

CD57, 20\% of the cases presented follicular T-cell rosettes, whereas with PD-1 (NAT-105), these were observed in $53 \%$ of cases. All cases with CD57positive T-cell rosettes also showed PD-1 positivity in the rosetting T-cells.

Staining with CD23 was performed in whole sections of 15 cases. In 13 of the 15 cases (86\%), tumoral cells were embedded in follicular dendritic cell meshworks, clearly surrounding the tumoral cells and forming nodular areas.

Statistical analysis revealed significant differences among the three diagnostic groups for many immunomarkers (Table 3).

\section{Reactive follicles}

Reactive tonsils and lymph nodes were analyzed as controls. Immunostaining showed the presence of large cells, positive for each of CD30, OCT.2, and BOB.1, which were limited to the outer zone of the germinal centers (Figure 4). Bcl-6 was not consistently expressed by the CD30-positive cells located in the outer zone.

Double immunofluorescence and immunohistochemical study

Reactive tonsils and lymph nodes showed CD30positive cells limited to the outer zone of the germinal centers partially surrounded by $\mathrm{T}$ cells, sometimes forming a perfect rosette, with an immunophenotype similar to the T-cell rosettes described in lymphocyte-rich classical Hodgkin's lymphoma and nodular lymphocyte-predominant Hodgkin's lymphoma, namely, CD57- and PD-1positive cases, respectively (Figure 4). This could also be seen in preserved residual germinal centers of six lymphocyte-rich classical Hodgkin's lymphoma samples, in which CD30-positive cells were also surrounded by $\mathrm{T}$ cells (PD-1-positive), and seemed to invade from the outer zone the interfollicular areas forming the tumoral mononuclear Hodgkin's cells and multinucleated Reed-Sternberg cells, still surrounded by $\mathrm{T}$ cells (Figure 5).

\section{Discussion}

Lymphocyte-rich classical Hodgkin's lymphoma has been described as being a distinct subtype of classical Hodgkin's lymphoma with scattered CD30-positive and CD15-positive HRS cells on a nodular or diffuse background of small lymphocytes. Morphologically, it mimics nodular lymphocyte-predominant Hodgkin's lymphoma, but has an immunophenotype similar to that of classical Hodgkin's lymphoma., ${ }^{1,7}$ Before this description, cases with the same features were known as 'follicular Hodgkin lymphoma.' ${ }^{\prime 8}$ Lymphocyte-rich classical Hodgkin's lymphoma has been considered by some authors to be a distinct entity, exhibiting features of classical Hodgkin's lymphoma and nodular lymphocyte-predominant Hodgkin's lymphoma, but with a different pattern of clinical presentation and age and sex distributions from other classical Hodgkin's lymphomas, and an excellent prognosis after treatment with current treatment regimens. ${ }^{2}$ Conversely, other investigators have suggested that lymphocyterich classical Hodgkin's lymphoma represents an early stage in the spectrum of classical Hodgkin's lymphoma rather than being a biologically separate disease entity. ${ }^{9}$ The observations described here seem to confirm the distinct profile of lymphocyterich classical Hodgkin's lymphoma neoplastic cells and their microenvironment, with its characteristic mixture of features of nodular lymphocyte-predominant Hodgkin's lymphoma and classical Hodgkin's lymphoma. Thus, mononuclear Hodgkin's cells and multinucleated Reed-Sternberg cells in lymphocyte-rich classical Hodgkin's lymphoma exhibit a full classical Reed-Sternberg cell profile (CD30 positive, CD15 positive, EBV/LMP positive for EBV-positive cases) simultaneously with a relatively well-preserved B-cell transcription program including BOB.1, OCT.1, OCT.2, some GC markers such as KLHL6, a lower frequency of BCL6, and the same frequency of NF-kB activation markers as in classical Hodgkin's lymphoma. By contrast, nodular lymphocyte-predominant Hodgkin's lymphoma cases do not have a classical Reed-Sternberg cell profile. Most do not show NF-kB activation markers but instead display B-cell transcription machinery markers such as OCT.1, OCT.2, and BOB.1. The presence of B-cell transcription markers 


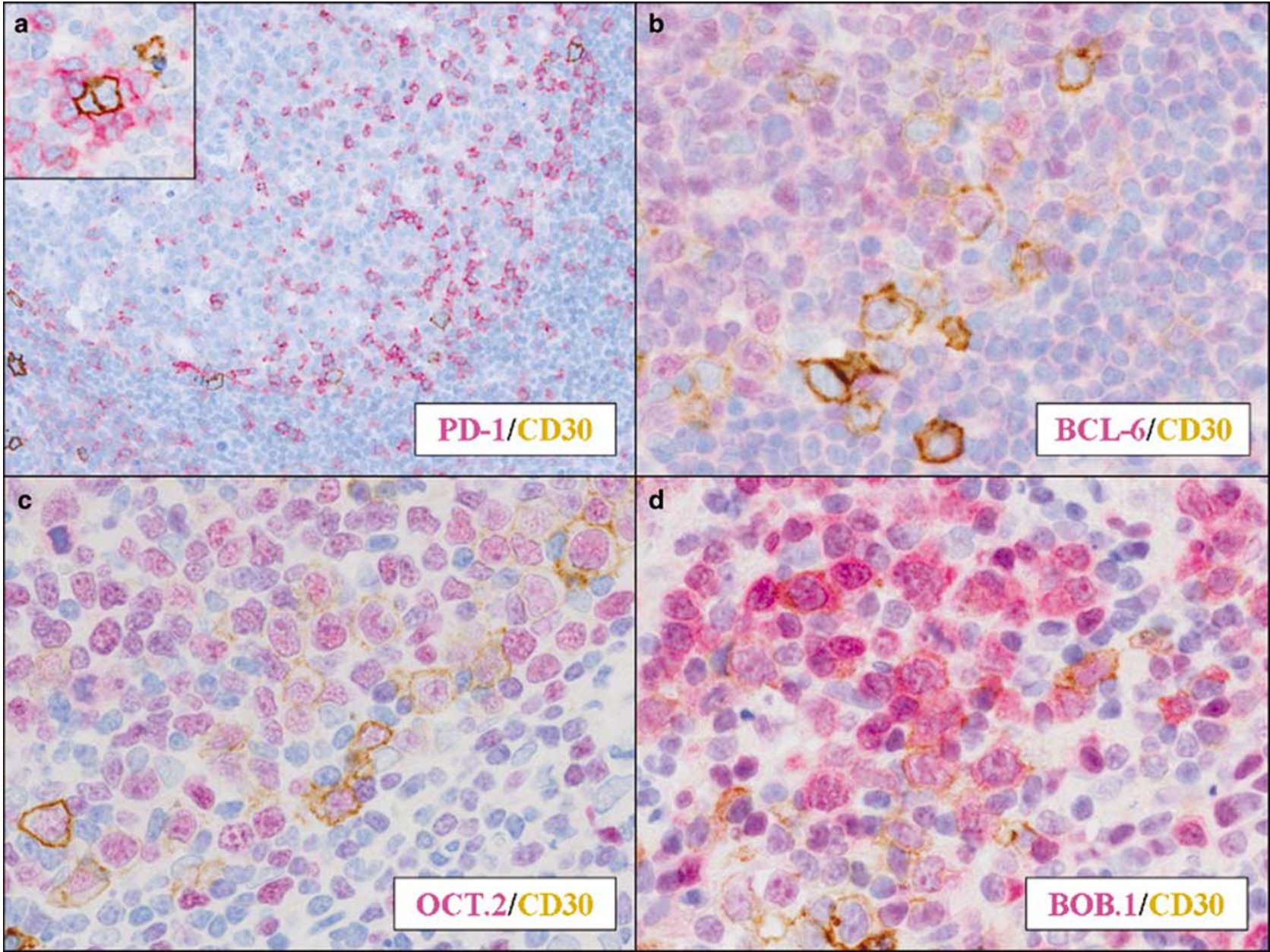

Figure 4 Reactive lymphoid follicles of the tonsil with the presence of CD30-positive cells surrounded by PD-1-positive T cells localized in the outer zone of the germinal center (a). These cells have a variable expression of Bcl-6 (b), and express OCT.2 (c) and BOB.1 (d).

in lymphocyte-rich classical Hodgkin's lymphoma is consistent with earlier observations made by Bräuninger et al., ${ }^{3}$ whose mutational studies of the rearranged Ig genes of mononuclear Hodgkin's cells and multinucleated Reed-Sternberg cells in lymphocyte-rich classical Hodgkin's lymphoma showed differences from those in lymphocytic and/or histiocytic Reed-Sternberg cell variants of nodular lymphocyte-predominant Hodgkin's lymphoma. Their presence also resembles the findings in classical Hodgkin's lymphoma, suggesting that mononuclear Hodgkin's cells and multinucleated Reed-Sternberg cells in lymphocyte-rich classical Hodgkin's lymphoma derive from germinal-center B-cells that silence hypermutation. ${ }^{3}$

Here we have analyzed relatively new germinalcenter markers such as KLHL6 and GCET1. KLHL6 is a BTB-kelch protein involved in germinal-center B-cell maturation, ${ }^{10}$ whereas GCET1 (germinal-center B cell-expressed transcript-1) is thought to be expressed exclusively by neoplasms that arrested at the germinal-center stage of differentiation. ${ }^{11}$ These markers were positive in most of the nodular lymphocyte-predominant Hodgkin's lymphoma cases, which is evidence of a germinal-center stage of differentiation in lymphocytic and/or histiocytic Reed-Sternberg cell variants. ${ }^{12}$ Interestingly, mononuclear Hodgkin's cells and multinucleated ReedSternberg cells in lymphocyte-rich classical Hodgkin's lymphoma commonly express KLHL6 (69\%, 11 of 16), a molecule that has been implicated in BCR signal transduction and in the formation of the full germinal-center response. ${ }^{10}$

$\mathrm{NF}-\mathrm{\kappa B}$ activation is considered to be an important survival factor for mononuclear Hodgkin's cells and multinucleated Reed-Sternberg cells in classical Hodgkin's lymphoma, whereby the inactivation of factor NF-אB in Hodgkin's lymphoma cell lines leads to the apoptosis of neoplastic cells. ${ }^{13,14}$ Different expression patterns of NF-kB subunits and related molecular factors have been shown in classical Hodgkin's lymphoma and nodular lymphocyte-predominant Hodgkin's lymphoma, with c-Rel protein, Rel-B, p-50, TRAF1, and MUM-1 being significantly more frequently expressed in the former. Our results are similar, supporting the 


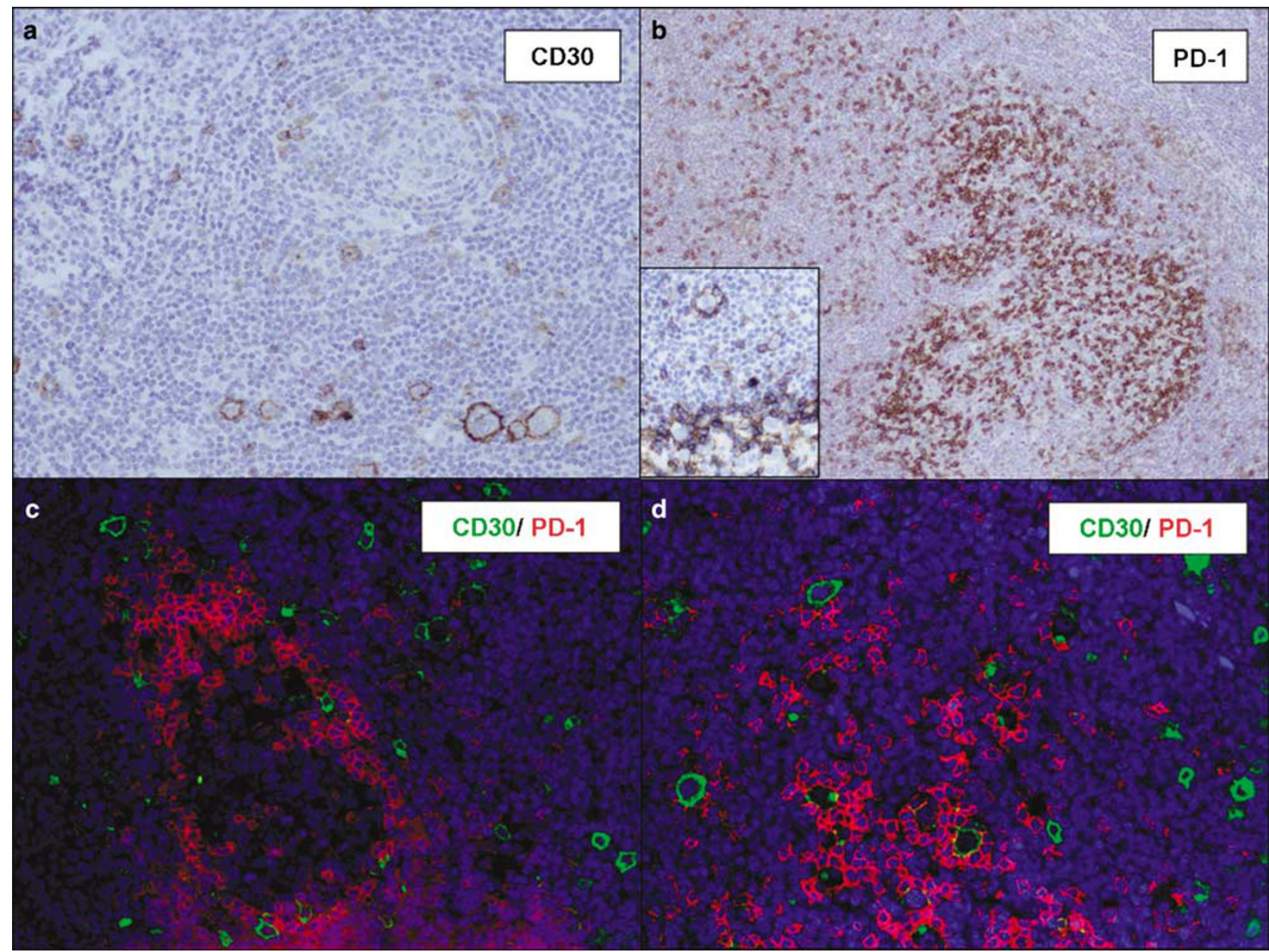

Figure 5 CD30-positive cells localized in the outer zone of residual germinal centers of LRCHL cases (a) surrounded by PD-1-positive T cells (b). Also evidenced by the double immunofluorescence study (c, d).

hypothesis that there is a low or null degree of NF$\kappa \mathrm{B}$ activation in nodular lymphocyte-predominant Hodgkin's lymphoma. Interestingly, lymphocyterich classical Hodgkin's lymphoma not only resembles nodular lymphocyte-predominant Hodgkin's lymphoma in its low level of Rel-B and C-Rel expression but also resembles classical Hodgkin's lymphoma with its high degree of expression of TRAF1 and p-50, ${ }^{15,16}$ which supports the interpretation that NF- $\mathrm{KB}$ is activated only by canonical or classical pathways, and not by non-canonical or alternative pathways in lymphocyte-rich classical Hodgkin's lymphoma.

With regard to the microenvironment, we have examined the presence of follicular T-cell rosettes with preserved follicular dendritic cell meshworks. The presence and histological distribution of follicular T cells is becoming more widely considered as useful for exploring the pathogenesis of several lymphoproliferative disorders. ${ }^{5,17,18}$ Follicular T-cell rosettes surrounding tumoral lymphocytic and/or histiocytic Reed-Sternberg cell variants within nodular areas of follicular dendritic cell meshworks are considered a diagnostic feature of nodular lymphocyte-predominant Hodgkin's lymphoma. These cells have an immunophenotype similar to that of intrafollicular T cells, that is, they are CD57 positive and PD- 1 positive and co-express bcl-6. ${ }^{19}$ We recently found that the presence of T-cell rosettes is not a unique and defining feature of nodular lymphocyte-predominant Hodgkin's lymphoma, as it also occurs in lymphocyte-rich classical Hodgkin's lymphoma. ${ }^{17}$ None of the cases of classical Hodgkin's lymphoma in this study presented T-cell rosettes, but a significantly large number of lymphocyte-rich classical Hodgkin's lymphoma cases showed follicular T-cell rosettes, presumably reflecting the germinal-center origin of the tumoral cells in nodular lymphocyte-predominant Hodgkin's lymphomas, and in at least a substantial proportion of lymphocyte-rich classical Hodgkin's lymphomas. ${ }^{17}$ These rosettes had already been recognized by Ashton-Key et al. ${ }^{8}$ in a series of four cases of CD30-positive and CD15-positive classical Hodgkin's lymphomas that histologically resembled nodular lymphocyte-predominant Hodgkin's 
lymphoma, although the follicular T-cell phenotype of the rosetting cells was not noted. The immunophenotype of the tumoral lymphocytic and/or histiocytic Reed-Sternberg cell variants in nodular lymphocyte-predominant Hodgkin's lymphoma (CD20, OCT.2, BOB.1, BCL6, and PAX-5) shows that nodular lymphocyte-predominant Hodgkin's lymphoma cells preserve the expression of a full B-cell program, whereas the presence of follicular $\mathrm{T}$ cells surrounding tumoral cells suggests that the germinal-center microenvironment is also maintained. This is not the case for classical Hodgkin's lymphoma, in which the B-cell program and the germinal-center microenvironment are lost. Nevertheless, in lymphocyte-rich classical Hodgkin's lymphoma, the B-cell program and the germinalcenter microenvironment seem to be at least partially preserved.

Neoplastic cell features and microenvironment sub-populations in lymphocyte-rich classical Hodgkin's lymphoma bear a resemblance to the CD30positive large B-cells situated in the outer zone of reactive germinal centers that are present in tonsils and lymphadenitis, and also in the residual germinal centers of lymphocyte-rich classical Hodgkin's lymphoma-involved lymph nodes. The outer zone refers to the germinal-center border adjacent to the follicular mantle. The outer zone of the follicle, first described by Hardie et al.,$^{20}$ is distinguished by the presence of follicular dendritic cells with a low level of expression of CD23, which distinguishes it from the adjacent follicular mantle and apical light zone. The outer zone was described as containing small lymphoid cells, blasts, and plasmacytoid cells, with many cells of the outer zone strongly expressing CDw75. Hauser et al. ${ }^{21}$ described the tendency of germinal-center B cells to migrate toward the outer zone of the germinal center, and Brink ${ }^{22}$ questioned whether this reflected a need for interactions with $\mathrm{T}$ cells or antigen-bearing cells bordering the germinal center. Indeed, although the specific function of the outer zone is still essentially unknown, antibodies for follicular T-cell markers such as PD-1 clearly show that the PD-1-positive cells in reactive follicles very characteristically occupy this outer zone. ${ }^{17}$ Interestingly, B-cell blasts situated in the outer zone have an immunophenotype that closely mimics that of lymphocyte-rich classical Hodgkin's lymphoma neoplastic cells, with expressions of OCT.2 and BOB.1, and a lower frequency of BCL6. These B-cell blasts express CD30 and are surrounded by a rim of follicular T cells.

In conclusion, lymphocyte-rich classical Hodgkin's lymphoma seems to be a distinct entity that shares similarities with both classical Hodgkin's lymphoma and nodular lymphocyte-predominant Hodgkin's lymphoma, and seems to derive from germinal-center $\mathrm{B}$ cells that maintain a germinalcenter microenvironment, mimicking the patterns seen in B-cell blasts and T-cell sub-populations in the outer zone of the reactive germinal centers.

\section{Acknowledgements}

We are grateful to the pathology, hematology, and oncology departments of all the Spanish hospitals who have provided us with the cases. We also express our appreciation to the staff of the CNIO Tumor Bank for their efficient provision of tumor samples. This study was supported by grants from the Ministerio de Sanidad y Consumo (Acción Transversal, RETICS, PI051623, PI052800), the Ministerio de Ciencia y Tecnología (SAF2005-00221, SAF2008-3871), and Fundación la Caixa, Spain.

\section{Disclosure/conflict of interest}

The authors declare no conflict of interest.

\section{References}

1 Jaffe ES. Pathology and genetics of tumours of haematopoietic and lymphoid tissues. IARC Press: Lyon, Washington, DC, 2001.

2 Shimabukuro-Vornhagen A, Haverkamp H, Engert A, et al. Lymphocyte-rich classical Hodgkin's lymphoma: clinical presentation and treatment outcome in 100 patients treated within German Hodgkin's Study Group trials. J Clin Oncol 2005;23:5739-5745.

3 Brauninger A, Wacker HH, Rajewsky K, et al. Typing the histogenetic origin of the tumor cells of lymphocyte-rich classical Hodgkin's lymphoma in relation to tumor cells of classical and lymphocyte-predominance Hodgkin's lymphoma. Cancer Res 2003;63:1644-1651.

4 Garcia-Cosio M, Santon A, Martin P, et al. Analysis of transcription factor OCT.1, OCT.2 and BOB.1 expression using tissue arrays in classical Hodgkin's lymphoma. Mod Pathol 2004;17:1531-1538.

5 Roncador G, Garcia Verdes-Montenegro JF, Tedoldi S, et al. Expression of two markers of germinal center $\mathrm{T}$ cells (SAP and PD-1) in angioimmunoblastic T-cell lymphoma. Haematologica 2007;92:1059-1066.

6 Mason DY, Micklem K, Jones M. Double immunofluorescence labelling of routinely processed paraffin sections. J Pathol 2000;191:452-461.

7 Diehl V, Sextro M, Franklin J, et al. Clinical presentation, course, and prognostic factors in lymphocytepredominant Hodgkin's disease and lymphocyte-rich classical Hodgkin's disease: report from the European Task Force on Lymphoma Project on LymphocytePredominant Hodgkin's Disease. J Clin Oncol 1999;17:776-783.

8 Ashton-Key M, Thorpe PA, Allen JP, et al. Follicular Hodgkin's disease. Am J Surg Pathol 1995;19: 1294-1299.

9 de Jong D, Bosq J, MacLennan KA, et al. Lymphocyterich classical Hodgkin lymphoma (LRCHL): clinicopathological characteristics and outcome of a rare entity. Ann Oncol 2006;17:141-145.

10 Kroll J, Shi X, Caprioli A, et al. The BTB-kelch protein KLHL6 is involved in B-lymphocyte antigen receptor signaling and germinal center formation. Mol Cell Biol 2005;25:8531-8540.

11 Montes-Moreno S, Roncador G, Maestre L, et al. Gcet1 (centerin), a highly restricted marker for a subset of 
germinal center-derived lymphomas. Blood 2008;111: 351-358.

12 Chan WC. Cellular origin of nodular lymphocytepredominant Hodgkin's lymphoma: immunophenotypic and molecular studies. Semin Hematol 1999;36: 242-252.

13 Hinz M, Loser P, Mathas S, et al. Constitutive NFkappaB maintains high expression of a characteristic gene network, including CD40, CD86, and a set of antiapoptotic genes in Hodgkin/Reed-Sternberg cells. Blood 2001;97:2798-2807.

14 Izban KF, Ergin M, Huang Q, et al. Characterization of NF-kappaB expression in Hodgkin's disease: inhibition of constitutively expressed NF-kappaB results in spontaneous caspase-independent apoptosis in Hodgkin and Reed-Sternberg cells. Mod Pathol 2001;14:297-310.

15 Xiao Q, Shen N, Hedvat CV, et al. Differential expression patterns of c-REL protein in classic and nodular lymphocyte predominant Hodgkin lymphoma. Appl Immunohistochem Mol Morphol 2004;12:211-215.

16 Rodig SJ, Savage KJ, Nguyen V, et al. TRAF1 expression and c-Rel activation are useful adjuncts in distinguishing classical Hodgkin lymphoma from a subset of morphologically or immunophenotypically similar lymphomas. Am J Surg Pathol 2005;29: 196-203.

17 Nam-Cha SH, Roncador G, Sanchez-Verde L, et al. PD1, a follicular T-cell marker useful for recognizing nodular lymphocyte-predominant Hodgkin lymphoma. Am J Surg Pathol 2008;32:1252-1257.

18 Vinuesa CG, Tangye SG, Moser B, et al. Follicular B helper $\mathrm{T}$ cells in antibody responses and autoimmunity. Nat Rev Immunol 2005;5:853-865.

19 Kraus MD, Haley J. Lymphocyte predominance Hodgkin's disease: the use of bcl-6 and CD57 in diagnosis and differential diagnosis. Am J Surg Pathol 2000;24:1068-1078.

20 Hardie DL, Johnson GD, Khan M, et al. Quantitative analysis of molecules which distinguish functional compartments within germinal centers. Eur J Immunol 1993;23:997-1004.

21 Hauser AE, Junt T, Mempel TR, et al. Definition of germinal-center $\mathrm{B}$ cell migration in vivo reveals predominant intrazonal circulation patterns. Immunity 2007;26:655-667.

22 Brink R. Germinal-center B cells in the zone. Immunity 2007;26:552-554. 